\title{
Maternal pre-pregnancy body mass index and fetal acidosis in vaginal deliveries and cesarean deliveries: The Japan Environment and Children's Study
}

\author{
Tsuyoshi Murata ${ }^{1}$, Hyo Kyozuka ${ }^{2}$, Akiko Yamaguchi ${ }^{2}$, Toma Fukuda ${ }^{2}$, Shun Yasuda ${ }^{2}$, \\ Akiko Sato $^{2}$, Yuka Ogata ${ }^{2}$, Masahito Kuse², Mitsuaki Hosoya ${ }^{2}$, Seiji Yasumura ${ }^{2}$, Koichi \\ Hashimoto $^{2}$, Hidekazu Nishigori ${ }^{2}$, and Keiya Fujimori ${ }^{2}$ \\ ${ }^{1}$ Fukushima Medical University School of Medicine Department of Obstetrics and \\ Gynecology \\ ${ }^{2}$ Fukushima Medical University
}

May 28, 2020

\begin{abstract}
Objective: To evaluate the association between maternal pre-pregnancy body mass index and fetal acidosis while accounting for the mode of delivery. Design: Prospective cohort study Setting: Japan Population: Participants from the Japan Environment and Children's Study with singleton pregnancies after 22 weeks of gestation who gave birth during 2011-2014 Methods: Participants were categorized into five groups according to pre-pregnancy body mass index $(\mathrm{BMI})(\mathrm{kg} / \mathrm{m} 2): \mathrm{G} 1(\mathrm{BMI}<18.5)$, G2 (18.5 to <20.0), G3 (20.0 to <23.0), G4 (23.0 to <25.0), and G5 ([?]25.0). Multiple logistic regression analysis evaluated the effect of maternal pre-pregnancy BMI on fetal acidosis while accounting for the mode of delivery. Main outcome measures: Fetal acidosis was defined as umbilical artery $\mathrm{pH}(\mathrm{UmA}-\mathrm{pH})<7.2$ or $<7.1$. Results: We analyzed 71,799 participants. Adjusted odds ratios (aORs) of UmA-pH $<7.2$ using G3 as the reference group were 1.17 (95\% confidence interval [CI], 1.06-1.30) in G5 and 0.89 (95\% CI, 0.82-0.97) in G2. After stratification, aORs of UmA-pH <7.2 were 1.12 (95\% CI, 1.08-1.35) in G5 and 0.90 (95\% CI, 0.83-0.98) in G2, and the aOR of UmA-pH <7.1 was 0.80 (95\% CI, 0.65-0.98) in G2 using G3 as the reference group for vaginal delivery. No association existed between pre-pregnancy BMI and fetal acidosis for delivery via cesarean section. Conclusions: In Japanese women, pre-pregnancy BMI [?]25.0 kg/m2 significantly increased the likelihood of fetal acidosis in newborns delivered vaginally.
\end{abstract}

\section{Hosted file}

BMI manuscript final BJOG.doc available at https://authorea.com/users/327152/articles/454816maternal-pre-pregnancy-body-mass-index-and-fetal-acidosis-in-vaginal-deliveries-andcesarean-deliveries-the-japan-environment-and-children-s-study

\section{Hosted file}

Figure 1.docx available at https://authorea.com/users/327152/articles/454816-maternalpre-pregnancy-body-mass-index-and-fetal-acidosis-in-vaginal-deliveries-and-cesareandeliveries-the-japan-environment-and-children-s-study 